\title{
Tumor Location of the Lower-Inner Quadrant Is Associated with an Impaired Survival for Women With Early-Stage Breast Cancer
}

\author{
Séverine Sarp, MD, ${ }^{1}$ Gérald Fioretta, BSc, ${ }^{1}$ Helena M. Verkooijen, MD, PhD, ${ }^{1}$ \\ Georges Vlastos, MD, ${ }^{2}$ Elisabetta Rapiti, MD, MPH, ${ }^{1}$ Hyma Schubert, MA, ${ }^{1}$ \\ André-Pascal Sappino, $\mathrm{MD},{ }^{3}$ and Christine Bouchardy, $\mathrm{MD}, \mathrm{PhD}^{1}$
}

\footnotetext{
${ }^{1}$ Geneva Cancer Registry, Institute for Social and Preventive Medicine, University of Geneva, Geneva, Switzerland ${ }^{2}$ Senology and Surgical Gynecologic Oncology Unit, Department of Gynecology and Obstetrics, Geneva University Hospitals, Geneva, Switzerland

${ }^{3}$ Division of Oncology, Department of Medicine, Geneva University Hospitals, Geneva, Switzerland
}

\begin{abstract}
Background: There is growing evidence that tumors of the inner quadrants (especially the lower-inner quadrant) metastasize more often to the internal mammary chain (IMC). As these metastases are not investigated, patients with lower-inner quadrant tumors have an increased risk of being under-staged and under-treated and may therefore have a higher risk of death from breast cancer.

Methods: We identified all 1522 women operated for stage I breast cancer between 1984 and 2002 recorded at the population-based Geneva Cancer Registry. We compared breast cancer mortality risk by tumor location with multivariate Cox regression analysis that accounted for all factors linked to tumor location and survival.

Results: Ten-year disease-specific survival was 93\% (95\%CI: 91-94\%). Patients with breast cancer of the lower-inner quadrant $(\mathrm{n}=118 ; 7.8 \%)$ had an importantly increased risk of dying of breast cancer compared to women with breast cancer of the upper-outer quadrant (multiadjusted Hazard Ratio: 2.3, 95\%CI: 1.1-4.5, $P=0.0206$ ). The over-mortality associated with this quadrant was particularly evident for tumors $>10 \mathrm{~mm}$ (multiadjusted HR: 3.6, 95\%CI: $1.6-7.9, P=0.0016)$. There was no increased breast cancer mortality risk for tumors located in other quadrants.

Conclusions: Tumor location in the lower-inner quadrant is an independent and important prognostic factor of stage I breast cancer. Further research is needed to evaluate if the overmortality of patients with stage I cancer of the lower-inner quadrant is indeed a result of under-treatment due to undetected IMC metastases. If so, patients with stage I breast cancer of the lower-inner quadrant are good candidates for systematic IMC investigation.
\end{abstract}

Key Words: Breast cancer-Early-stage - Population-based-Survival_-Tumor location.

Received July 20, 2006; accepted August 29, 2006; published online December 20, 2006.

Part of this study was presented as a poster at the $28^{\text {th }}$ San Antonio Breast Cancer Symposium, December 8-11, 2005.

Address correspondence and reprint requests to: Christine Bouchardy, MD, PhD, Geneva Cancer Registry, 55 Boulevard de la Cluse, 1205 Geneva, Switzerland; E-mail: christine.bouchardymagnin@imsp.unige.ch

Published by Springer Science+Business Media, Inc. @ 2006 The Society of Surgical Oncology, Inc.
With the generalization of mammography screening, many breast cancers are detected at an early stage. Women diagnosed with tumors of $\leq 20 \mathrm{~mm}$, without lymph node involvement and distant metastases (T1 N0 M0, stage I), have a five-year diseasespecific survival of approximately $98 \% .{ }^{1}$ Despite this excellent general prognosis, some patients with early stage breast cancer die of their disease. These deaths are often related to poor tumor differentiation, 
absence of hormonal receptors, or suboptimal treatment. ${ }^{2,3}$ Erroneous staging could also be a reason for some of these breast cancer deaths.

Veronesi and colleagues performed a systematic surgical evaluation of the internal mammary chain (IMC) and found that approximately $10 \%$ of breast cancers without axillary lymph node involvement metastasize to the IMC. ${ }^{4}$ This risk of IMC involvement increases with tumor size. ${ }^{4,5}$ In addition, the presence of IMC metastases depends on tumor location in the breast, with a higher prevalence of IMC metastases in tumors of the inner quadrants, especially the lower-inner quadrant..$^{6-8}$

The IMC is not routinely investigated for the presence of lymph node metastases, and breast cancer is classified as lymph node negative on the basis of axillary lymph node investigations only. Undetected IMC metastases, therefore, lead to under-staging of patients without axillary lymph node involvement and to under-treatment of stage I patients who have no indication of adjuvant chemotherapy. There is growing evidence that tumors occurring in the inner quadrants have a higher breast cancer mortality. ${ }^{9-12}$ However, the real impact of tumor location on the risk of IMC node metastases and on prognosis is still controversial, mainly because of the inconclusive findings in older studies. ${ }^{4,13-15}$

We used data from the Geneva Cancer Registry to evaluate the impact of tumor location on breast cancer mortality among women with stage I disease.

\section{PATIENTS AND METHODS}

The Geneva Cancer Registry records all incident cancer cases occurring in the population of the canton since 1970. Registration is based on various sources of information and can be considered as accurate, as attested by its low percentage (2\%) of cases recorded from death certificates only. Every public hospital, private clinic, pathology laboratory, and physician is requested to report all cancer cases. Individual clinical files from the university public hospitals are systematically consulted and inquiry forms are sent to physicians for patients treated in the private sector. The registry actively assesses survival by an annual, systematic check of vital status in the files of the Cantonal Population Office and passively by routine examination of hospital records and death certificates. The cause of death is systematically recorded and validated. Recorded data include sociodemographic characteristics, method of discovery, tumor characteristics (coded according to the Inter- national Classification of Diseases for Oncology [ICD-O]), stage (according to tumor, lymph node, and metastasis classification TNM), treatment during the first six months after diagnosis, healthcare sector, and date and cause of death (coded according to the World Health Organization's classification).

We selected all women operated between 1984 and $2002(\mathrm{n}=1695)$ for invasive stage I breast cancer, defined as pathological pT1 $(\leq 2 \mathrm{~cm}$ ), pN0 (no lymph node invasion), and without distant metastases. We excluded women with other breast cancers $(\mathrm{n}=108)$ or other invasive cancers (except non-melanoma skin cancer) $(n=65)$ before or within six months after their breast cancer diagnosis. The study finally included 1522 women.

Variables of interest included age (in continuous), period of diagnosis (1984-1989; 1990-1994; 19951999; 2000-2002), method of discovery (symptoms or fortuitous, i.e. diagnosis during the work-up of an unrelated illness, breast self-examination, and screening), civil status (single, married, widowed, and divorced/separated), place of birth (Switzerland, Southern Europe, and other), socioeconomic status based on the woman's last occupation (high, middle, low, unknown), and healthcare sector in charge of breast cancer surgery (private clinics or public university hospitals).

Histologic types were regrouped as ductal (ICD-O codes 8010, 8140, and 8500), lobular (ICD-O codes 8520 and 8522), mucinous (ICD-O codes 8480 and 8481 ), and other. Tumor differentiation was classified as good (grade I), moderate (grade II), poor (grade III), or unknown. Estrogen receptor status was classified as negative, positive (when $\geq 10 \%$ of cells expressed estrogen receptors), or unknown. Tumor size was regrouped as 1-10 mm, 11-20 mm, or unknown. IMC lymph node status was not systematically assessed during the study period in Geneva and the Geneva Cancer Registry has no information on IMC lymph node involvement. Surgery was classified as mastectomy, surgery with positive margins, and breast-conserving surgery. Radiotherapy was considered as yes vs. no. Systemic treatment was categorized as chemotherapy (yes, no) and hormone therapy (yes, no).

We considered the following anatomical breast locations: upper-outer quadrant (ICD-O codes C50.4, C50.6), upper-inner quadrant (ICD-O code C50.2), lower-outer quadrant (ICD-O code C50.5), lower-inner quadrant (ICD-O code 50.3), nipple and central portion of the breast (ICD-O codes C50.0, C50.1), and overlapping quadrant (ICD-O code C50.8), and unknown (ICD-O code C50.9). 
Associations between tumor location and sociodemographic, tumor and treatment characteristics were evaluated by chi-square test. With multivariate logistic regression analysis, we identified factors independently and significantly linked to tumor location. ${ }^{16}$ Breast cancer-specific survival was calculated using the actuarial method, considering death from breast cancer only. We applied the log-rank test to evaluate differences in survival curves. By means of univariate Cox proportional hazards analysis, we identified all variables significantly associated with breast cancer mortality. With multivariate analysis, adjusting for all variables significantly associated with breast cancer mortality or tumor location, we estimated the effect of tumor location on breast cancer mortality.

Because larger tumors are more prone to metastasize, we tested for the interaction between tumor location and size to investigate if the effect of location was similar for larger (11-20 mm) and smaller tumors $(1-10 \mathrm{~mm}) .{ }^{16}$ Moreover, because chemotherapy decreases the higher mortality associated with positive lymph nodes, we also tested for interaction between tumor location and use of adjuvant chemotherapy. Statistical analyses were performed with SPSS software (SPSS 11.5 version Inc., Chicago, IL).

\section{RESULTS}

Among the 1522 patients operated for stage I breast cancer, the most common tumor location was the upper-outer quadrant (39\%), followed by lesions located in overlapping quadrants $(27 \%)$, the upperinner quadrant (14\%), the lower-outer quadrant $(8 \%)$, the lower-inner quadrant $(8 \%)$, and the central portion of the breast and the nipple (4\%). The median follow-up was 7.7 years and 472 patients (31\%) had a follow-up of 10 years or more. During the study period, 81 patients $(5 \%)$ died as a result of breast cancer and 130 patients $(8 \%)$ were lost to follow-up as they had left Geneva for another canton or country. Ten-year specific survival was $93 \%$ (95\% CI: 91-94\%).

Breast cancer-specific survival curves according to tumor location are presented in Fig. 1. The 10-year specific survival was 94\% (95\%CI: 91-96\%) for patients with cancer of the upper-outer quadrant, $96 \%$ (95\%CI: 92-99\%) for the upper-inner quadrant, 91\% (95\% C: $85-98 \%)$ for the lower-outer quadrant, 94\% (95\%CI: $87-100 \%)$ for the central part of the breast and the nipple, and 93\% (95\% CI: 89-96\%) for overlapping lesions. For patients with tumors of the lower-inner quadrant, however, the 10-year disease specific survival was significantly lower, $88 \%$ (95\% CI: $81-96 \%, P$ log-rank test 0.0002 ).

Table 1 shows that tumors of the lower-inner quadrant were less often detected by screening $(P=$ 0.011 ), were less frequently of lobular histology $(P=0.014)$, were more often poorly differentiated $(P=0.031)$, had less frequently breast conserving surgery and more frequently mastectomy $(P=0.003)$, and received less often hormone therapy $(P=0.031)$. In the multiadjusted logistic regression, only histology, poor differentiation, and type of surgery remained significantly and independently associated with tumor location in the lower-inner quadrant.

Tables 2 and 3 present the effect of patient and tumor characteristics and treatments on breast cancer mortality. In the multivariate model, we included all variables that were significantly associated with mortality in univariate analysis (i.e. age, period, method of discovery, grade, tumor size, surgery, radiotherapy, tumor location), factors significantly associated with tumor location (i.e. method of discovery, histologic type, grade, type of surgery, and hormone therapy), period of diagnosis, and age.

Table 4 presents the impact of tumor location on breast cancer mortality. In multiadjusted Cox proportional hazard analysis, tumor location remains a significant prognostic factor. Women with tumors located in the lower-inner quadrant of the breast had a more than two-fold increased risk of dying as a result of breast cancer compared with women with tumors of the upper-outer quadrant (multi-adjusted HR: 2.3 , 95\%CI: $1.1-4.5, P=0.021$ ). Tumor location in other quadrants was not associated with a significantly altered mortality risk.

The effect of lower-inner location appeared to be more obvious in patients with tumors larger than $10 \mathrm{~mm}(\mathrm{n}=1001$, multi-adjusted HR: 3.6, 95\%CI: $1.6-7.9, P=0.002)$ than among patients with smaller tumors $(\mathrm{n}=491$, age adjusted HR: 1.0, 95\% CI: $0.2-4.6, P=0.970)$ and in patients who did not receive adjuvant chemotherapy $(\mathrm{n}=1,261$, multiadjusted HR: $2.5,95 \% \mathrm{CI}: 1.2-5.4, P=0.016)$ than in patients who underwent chemotherapy $(\mathrm{n}=261$, ageadjusted HR: $1.6,95 \% \mathrm{CI}: 0.2-13.2, P=0.662$ ). However, none of the interaction tests between location and size or between location and chemotherapy were statistically significant $(P=0.134$ and $P=0.913$, respectively). Since we observed only few deaths $(\mathrm{n}=20)$ among patients with tumors $\leq 10 \mathrm{~mm}$ and among patients with chemotherapy $(\mathrm{n}=15)$, we adjusted the hazard ratio's only for age in these subgroup analyses. 


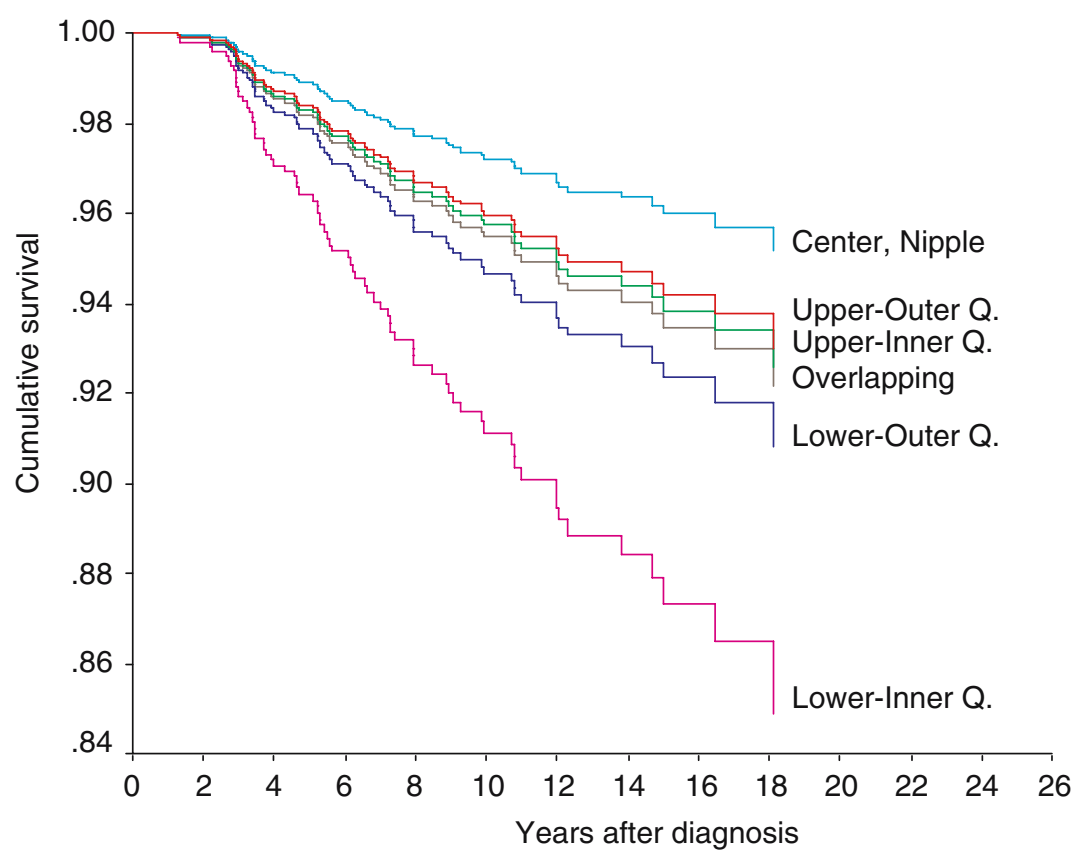

Number of women at risk at the beginning of each period

$\begin{array}{lrrrrr}\text { Year } & \text { 1st } & \text { 5th } & \text { 10th } & \text { 15th } & \text { 18th } \\ \text { Center, nipple } & 54 & 36 & 25 & 10 & 4 \\ \text { Upper-outer } & 588 & 424 & 217 & 74 & 30 \\ \text { Upper-inner } & 212 & 137 & 70 & 27 & 11 \\ \text { Overlapping } & 413 & 283 & 137 & 55 & 24 \\ \text { Lower-outer } & 118 & 82 & 43 & 24 & 9 \\ \text { Lower-inner } & 118 & 84 & 45 & 18 & 6\end{array}$

${ }^{\text {a }}$ Survival curves derived from Cox model adjusted for age (variable in continuous), period of diagnosis, method of discovery, grade, tumor size, surgery, radiotherapy, tumor location, histologic type and hormone therapy; Only deaths from breast cancer are considered.

FIG. 1. Specific survival curves ${ }^{\mathrm{a}}$ according to tumor location among women operated for stage 1 breast cancer, Geneva Cancer Registry, 1984-2002.

\section{DISCUSSION}

This study shows that tumor location within the breast is an important and independent prognostic factor of early-stage breast cancer. After adjustment for all factors linked to tumor location or prognosis, patients with tumors of the lower-inner quadrant have a more than two-fold increased risk of dying as a result of breast cancer compared to patients with tumors of the upper-outer quadrant. No difference in mortality from breast cancer was observed for the other tumor locations. Differences in tumor characteristics or treatment did not explain the over-mortality associated with the lower-inner quadrant. The results of this study also suggest that the impact of tumor location is more pronounced for women with larger tumors and for women who are not treated with adjuvant chemotherapy.

We acknowledge that we had information on various prognostic factors for breast cancer. However, for a relatively high proportion of patients (49\%), we had no information on estrogen receptor status as this information became available only after 1995 . When considering only patients diagnosed after this date $(\mathrm{n}=798$, proportion of patients with unknown receptor status: 3\%), we observed similar results (multiadjusted HR for tumors of the lower-inner quadrant compared to the upper-outer quadrant: 3.7 , 95\% CI: $0.7-18.7, P=0.110$ ). We also acknowledge that our study has limited power for subgroup analyses due to the relatively low number of breast cancer deaths occurring in patients with stage I breast cancer. 
TABLE 1. Patient and tumor characteristics according to tumor location among patients operated for stage I breast cancer, Geneva Cancer Registry, 1984-2002

\begin{tabular}{|c|c|c|c|}
\hline \multicolumn{4}{|c|}{ Tumor location } \\
\hline & Lower-inner quadrant & Other & $\begin{array}{l}P \text { value for } \mathrm{X}^{2} \text { of } \\
\text { heterogeneity }\end{array}$ \\
\hline Total & $\mathrm{n}=118(\%)$ & $\mathrm{N}=1404(\%)$ & \\
\hline \multicolumn{4}{|l|}{ Age group } \\
\hline$<40$ & $8(6.8)$ & $73(5.2)$ & 0.673 \\
\hline $40-49$ & $21(17.8)$ & $268(19.1)$ & \\
\hline $50-59$ & $31(26.3)$ & $450(32.1)$ & \\
\hline $60-69$ & $38(32.2)$ & $373(26.6)$ & \\
\hline $70-79$ & $17(14.4)$ & $196(14.0)$ & \\
\hline$\geq 80$ & $3(2.5)$ & $44(3.1)$ & \\
\hline \multicolumn{4}{|l|}{ Period of diagnosis } \\
\hline 1984-1989 & $38(32.2)$ & $313(22.3)$ & 0.100 \\
\hline 1990-1994 & $26(22.0)$ & $347(24.7)$ & \\
\hline 1995-1999 & $32(27.1)$ & $416(29.6)$ & \\
\hline $2000-2002$ & $22(18.6)$ & $328(23.4)$ & \\
\hline \multicolumn{4}{|l|}{ Method of discovery } \\
\hline Symptoms/fortuitous & $60(50.8)$ & $528(37.6)$ & 0.011 \\
\hline Self-examination & $22(18.6)$ & $274(19.5)$ & \\
\hline Screening & $36(30.5)$ & $602(42.9)$ & \\
\hline \multicolumn{4}{|l|}{ Histologic type } \\
\hline Ductal & 99 (83.9) & $1174(83.6)$ & 0.014 \\
\hline Lobular & $3(2.5)$ & $119(8.5)$ & \\
\hline Mucinous & $3(2.5)$ & $35(2.5)$ & \\
\hline Other & $13(11.0)$ & $76(5.4)$ & \\
\hline \multicolumn{4}{|l|}{ Grade } \\
\hline I & $30(25.4)$ & $529(37.7)$ & 0.031 \\
\hline II & $47(39.8)$ & $526(37.5)$ & \\
\hline III & $21(17.8)$ & $186(13.2)$ & \\
\hline Unknown & $20(16.9)$ & $163(11.6)$ & \\
\hline \multicolumn{4}{|l|}{ Tumor size } \\
\hline $1-10 \mathrm{~mm}$ & $37(31.4)$ & $454(32.3)$ & 0.947 \\
\hline $11-20 \mathrm{~mm}$ & $79(66.9)$ & $922(65.7)$ & \\
\hline Unknown & $2(1.7)$ & $28(2.0)$ & \\
\hline \multicolumn{4}{|l|}{ Estrogen receptor status } \\
\hline Negative & $8(6.8)$ & $95(6.8)$ & 0.254 \\
\hline Positive & $44(37.3)$ & $631(44.9)$ & \\
\hline Unknown & $66(55.9)$ & $678(48.3)$ & \\
\hline \multicolumn{4}{|l|}{ Surgery } \\
\hline Mastectomy & $38(32.2)$ & $361(25.7)$ & 0.003 \\
\hline Positive margins & $6(5.1)$ & $20(1.4)$ & \\
\hline Breast-conserving surgery & $74(62.7)$ & $1023(72.9)$ & \\
\hline \multicolumn{4}{|l|}{ Radiotherapy } \\
\hline Yes & $85(72.0)$ & $1066(75.9)$ & 0.344 \\
\hline No & $33(28.0)$ & $338(24.1)$ & \\
\hline \multicolumn{4}{|l|}{ Chemotherapy } \\
\hline Yes & $17(14.4)$ & $244(17.4)$ & 0.411 \\
\hline \multirow{2}{*}{\multicolumn{4}{|c|}{ Hormone therapy }} \\
\hline & & & \\
\hline Yes & $46(39.0)$ & $692(49.3)$ & 0.031 \\
\hline No & $72(61.0)$ & $712(50.7)$ & \\
\hline
\end{tabular}

Today, staging for breast cancer is mainly based on axillary lymph node status. Systematic IMC investigation stopped at the beginning of the 1980s after the publication of two important trials reporting no survival benefit for patients with negative axillary lymph nodes who underwent IMC dissection. ${ }^{5,17}$ However, none of these patients received adjuvant therapy, even if they had axillary or IMC lymph node metastases. This could explain the lack of efficacy of
IMC staging, as lymph node dissection is only a staging and not a curative procedure. ${ }^{5}$

Lymph node status, either axillary or internal mammary, is an important prognostic factor for patients with operable disease. ${ }^{17,18}$ Patients with IMC metastases have an impaired prognosis compared to patients with no mammary or axillary lymph node metastases (10-year survival of 53 vs. $80 \%$, respectively) and they have a similar survival as 
TABLE 2. Distribution and effect of sociodemographic characteristics, period of diagnosis and sector of care on breast cancer mortality among women operated for stage I breast cancer, Geneva Cancer Registry, 1984-2002

\begin{tabular}{|c|c|c|c|c|c|c|c|}
\hline \multirow[b]{2}{*}{ Patient characteristics } & \multicolumn{7}{|c|}{ Breast cancer mortality } \\
\hline & $\mathrm{N}$ & $\%$ & $\mathrm{~N}$ of death & $\%$ & 10-year survival $(95 \% \mathrm{CI})$ & Age-adjusted HR (95\%CI) & Multiadjusted $\mathrm{HR}^{a}(95 \% \mathrm{CI})$ \\
\hline \multicolumn{8}{|l|}{ Age group } \\
\hline$<40$ & 81 & 5.3 & 8 & 9.9 & $0.82(0.70-0.94)$ & $2.1^{b}(0.9-4.8)$ & $1.4^{b}(0.6-3.2)$ \\
\hline $40-49$ & 289 & 19.0 & 19 & 23.5 & $0.93(0.89-0.96)$ & $1.2^{b}(0.6-2.2)$ & $0.8^{b}(0.4-1.6)$ \\
\hline $50-59$ & 481 & 31.6 & 21 & 25.9 & $0.93(0.89-0.96)$ & 1 (reference) & 1 (reference) \\
\hline $60-69$ & 411 & 27.0 & 17 & 21.0 & $0.94(0.90-0.97)$ & $1.0^{b}(0.5-1.8)$ & $0.8^{b}(0.4-1.6)$ \\
\hline $70-79$ & 213 & 14.0 & 14 & 17.3 & $0.95(0.92-0.98)$ & $1.3^{b}(0.7-2.6)$ & $1.2^{b}(0.6-2.5)$ \\
\hline$\geq 80$ & 47 & 3.1 & 2 & 2.5 & $0.95(0.86-1.00)$ & $1.0^{b}(0.2-4.4)$ & $0.6^{b}(0.1-2.9)$ \\
\hline \multicolumn{8}{|l|}{ Period of diagnosis } \\
\hline 1984-1989 & 351 & 23.1 & 44 & 54.3 & $0.89(0.85-0.92)$ & 1 (reference) & 1 (reference) \\
\hline 1990-1994 & 373 & 24.5 & 22 & 27.2 & $0.95(0.92-0.97)$ & $0.6(0.3-1.0)$ & $0.9(0.5-1.6)$ \\
\hline 1995-1999 & 448 & 29.4 & 14 & 17.3 & $0.95^{c}(0.92-0.98)$ & $0.5(0.3-1.0)$ & $1.2(0.5-3.0)$ \\
\hline $2000-2002$ & 350 & 23.0 & 1 & 1.2 & $0.99^{d}(0.98-1.00)$ & $0.3(0.0-1.9)$ & $0.6(0.1-5.6)$ \\
\hline \multicolumn{8}{|l|}{ Method of discovery } \\
\hline Symptoms/fortuitous & 588 & 38.6 & 56 & 69.1 & $0.90(0.88-0.93)$ & 1 (reference) & 1 (reference) \\
\hline Breast self-examination & 296 & 19.4 & 6 & 7.4 & $0.95^{c}(0.90-1.00)$ & $0.5(0.2-1.2)$ & $0.6(0.2-1.8)$ \\
\hline Screening & 638 & 41.9 & 19 & 23.5 & $0.95(0.93-0.98)$ & $0.5^{*}(0.3-0.9)$ & $0.8(0.4-1.3)$ \\
\hline \multicolumn{8}{|l|}{ Civil status } \\
\hline Single & 186 & 12.2 & 12 & 14.8 & $0.91(0.85-0.97)$ & 1 (reference) & 1 (reference) \\
\hline Married & 916 & 60.2 & 51 & 63.0 & $0.92(0.90-0.94)$ & $0.8(0.4-1.5)$ & $1.0(0.5-1.9)$ \\
\hline Widowed & 211 & 13.9 & 8 & 9.9 & $0.97(0.94-1.00)$ & $0.6(0.2-1.4)$ & $0.7(0.2-1.7)$ \\
\hline Divorced, separated & 209 & 13.7 & 10 & 12.3 & $0.92(0.86-0.97)$ & $0.7(0.3-1.6)$ & $0.8(0.3-1.8)$ \\
\hline \multicolumn{8}{|l|}{ Place of birth } \\
\hline Switzerland & 816 & 53.6 & 45 & 55.6 & $0.93(0.91-0.95)$ & 1 (reference) & 1 (reference) \\
\hline Southern Europe & 386 & 25.4 & 21 & 25.9 & $0.93(0.89-0.96)$ & $1.0(0.6-1.7)$ & $0.9(0.5-1.6)$ \\
\hline Other & 320 & 21.0 & 15 & 18.5 & $0.92(0.87-0.96)$ & $1.1(0.6-1.9)$ & $1.1(0.6-2.0)$ \\
\hline \multicolumn{8}{|l|}{ Socioeconomic status } \\
\hline High & 204 & 13.4 & 10 & 12.3 & $0.94(0.90-0.98)$ & 1 (reference) & 1 (reference) \\
\hline Middle & 686 & 45.1 & 39 & 48.1 & $0.92(0.90-0.95)$ & $1.1(0.6-2.3)$ & $1.1(0.5-2.2)$ \\
\hline Low & 267 & 17.5 & 13 & 16.0 & $0.93(0.89-0.97)$ & $1.0(0.4-2.3)$ & $0.9(0.4-2.3)$ \\
\hline Unknown & 365 & 24.0 & 19 & 23.5 & $0.92(0.89-0.96)$ & $1.0(0.4-2.1)$ & $1.0(0.4-2.2)$ \\
\hline \multicolumn{8}{|l|}{ Sector of care } \\
\hline Private & 917 & 60.2 & 45 & 55.6 & $0.93(0.91-0.95)$ & 1 (reference) & 1 (reference) \\
\hline Public & 605 & 39.8 & 36 & 44.4 & $0.92(0.89-0.95)$ & $1.3(0.8-2.0)$ & $1.2(0.8-2.0)$ \\
\hline
\end{tabular}

${ }^{a}$ Hazard ratio adjusted for age (variable in continuous), period of diagnosis, method of discovery, grade, tumor size, surgery, radiotherapy, tumor location, histologic type, and hormone therapy; ${ }^{b}$ not adjusted for age, ${ }^{*} P<0.05, * * P<0.01,{ }^{* * *} P<0.001$; $^{c} 9$-year specific survival; ${ }^{d}$-year specific survival.

patients with axillary lymph node metastases. ${ }^{15}$ The efficacy of adjuvant chemotherapy to reduce mortality in patients with lymph node positive disease has been well established. ${ }^{19}$ Adjuvant radiotherapy of the IMC is still controversial and under investigation. ${ }^{20,21}$ Misclassification of patients as having lymph node negative disease has minor consequences if adjuvant chemotherapy is already indicated. For patients who have no indication for adjuvant chemotherapy, such under-staging results in undertreatment and probably in a higher risk of death from breast cancer.

IMC investigation is a rather invasive procedure, even with minimally invasive techniques. Are the available data sufficient to propose systematic IMC investigations for women who have no indication for adjuvant chemotherapy and present stage I breast cancer located in the lower-inner quadrant? In fact, there is growing evidence that the lower-inner quad- rant drains more often to the IMC than the other quadrants. ${ }^{8,22,23}$ In particular, Shahar and colleagues recently reported that $43 \%$ of the tumors of the lowerinner quadrant drains to the IMC compared to about $30 \%$ to the lower-outer quadrant or central portion, and approximately $15 \%$ of the other quadrants. ${ }^{8}$ Some authors, though, concluded that the risk of IMC metastases does not depend on the quadrant. In particular, the large study on extended radical mastectomy performed by Veronesi and colleagues ${ }^{4}$ showed an equal rate of IMC metastases for the inner and central quadrants $(20 \%)$ and the outer quadrants (16\%). Unfortunately, the 90 patients with tumors of the inner-lower were considered jointly with the 634 patients with tumors of the other inner and central quadrants. A reanalysis of this important series could therefore be particularly important to confirm whether IMC metastases are more frequent for cancers of the lower-inner quadrant or not. 
TABLE 3. Distribution and effect of tumor characteristics and treatment on breast cancer mortality among women operated for stage I breast cancer, Geneva Cancer Registry, 1984-2002

\begin{tabular}{|c|c|c|c|c|c|c|c|}
\hline \multirow{2}{*}{$\begin{array}{l}\text { Tumor characteristics } \\
\text { and treatment }\end{array}$} & \multicolumn{7}{|c|}{ Breast cancer mortality } \\
\hline & $\mathrm{N}$ & $\%$ & $\mathrm{~N}$ of death & $\%$ & 10-year survival $(95 \% \mathrm{CI})$ & Age-adjusted HR (95\%CI) & Multiadjusted $\mathrm{HR}^{a}(95 \% \mathrm{CI})$ \\
\hline \multicolumn{8}{|l|}{ Histologic type } \\
\hline Ductal & 1273 & 83.6 & 71 & 87.7 & $0.92(0.90-0.94)$ & 1 (reference) & 1 (reference) \\
\hline Lobular & 122 & 8.0 & 3 & 3.7 & $0.94(0.88-1.00)$ & $0.5(0.2-1.7)$ & $0.6(0.2-2.0)$ \\
\hline Mucinous & 38 & 2.5 & 2 & 2.5 & $0.96(0.87-1.00)$ & $0.8(0.2-3.4)$ & $1.0(0.2-4.5)$ \\
\hline Other & 89 & 5.8 & 5 & 6.2 & $0.94(0.88-1.00)$ & $0.8(0.3-2.0)$ & $0.7(0.2-1.8)$ \\
\hline \multicolumn{8}{|l|}{ Grade } \\
\hline I & 559 & 36.7 & 12 & 14.8 & $0.97(0.94-0.99)$ & 1 (reference) & 1 (reference) \\
\hline II & 573 & 37.6 & 31 & 38.3 & $0.93(0.90-0.95)$ & $2.2 *(1.1-4.2)$ & $1.9(1.0-3.8)$ \\
\hline III & 207 & 13.6 & 19 & 23.5 & $0.86(0.80-0.92)$ & $3.9 * * *(1.9-8.0)$ & $3.5 * * *(1.7-7.4)$ \\
\hline Unknown & 183 & 12.0 & 19 & 23.5 & $0.91(0.86-0.95)$ & $2.6^{*}(1.2-5.4)$ & $1.6(0.7-3.7)$ \\
\hline \multicolumn{8}{|l|}{ Tumor size } \\
\hline $1-10 \mathrm{~mm}$ & 491 & 32.3 & 20 & 24.7 & $0.94(0.91-0.97)$ & $0.7(0.4-1.1)$ & $0.7(0.4-1.2)$ \\
\hline $11-20 \mathrm{~mm}$ & 1001 & 65.8 & 54 & 66.7 & $0.93(0.91-0.95)$ & 1 (reference) & 1 (reference) \\
\hline Unknown & 30 & 2.0 & 7 & 8.6 & $0.75(0.57-0.93)$ & $2.6 *(1.2-5.8)$ & $2.8 *(1.1-7.1)$ \\
\hline \multicolumn{8}{|l|}{ Estrogen receptors status } \\
\hline Negative & 103 & 6.8 & 5 & 6.2 & $0.92^{b}(0.86-0.99)$ & 1 (reference) & 1 (reference) \\
\hline Positive & 675 & 44.3 & 9 & 11.1 & $0.96^{b}(0.92-0.99)$ & $0.3(0.1-1.0)$ & $0.6(0.2-2.0)$ \\
\hline Unknown & 744 & 48.9 & 67 & 82.7 & $0.92(0.90-0.94)$ & $0.7(0.3-1.9)$ & $0.8(0.1-7.8)$ \\
\hline \multicolumn{8}{|l|}{ Surgery } \\
\hline Mastectomy & 399 & 26.2 & 37 & 45.7 & $0.90(0.87-0.94)$ & 1 (reference) & 1 (reference) \\
\hline Breast-conserving surgery & 1097 & 72.1 & 42 & 51.9 & $0.94(0.92-0.96)$ & $0.6 *(0.4-0.9)$ & $0.8(0.4-1.7)$ \\
\hline Positive margins & 26 & 1.7 & 2 & 2.5 & $0.94(0.83-1.00)$ & $3.4(0.8-14.4)$ & $4.0(0.8-19.4)$ \\
\hline \multicolumn{8}{|l|}{ Radiotherapy } \\
\hline Yes & 1151 & 75.6 & 48 & 59.3 & $0.93(0.91-0.95)$ & 1 (reference) & 1 (reference) \\
\hline No & 371 & 24.4 & 33 & 40.7 & $0.92(0.88-0.95)$ & $1.6 *(1.0-2.5)$ & $1.1(0.5-2.3)$ \\
\hline \multicolumn{8}{|l|}{ Chemotherapy } \\
\hline Yes & 261 & 17.1 & 15 & 18.5 & $0.90(0.84-0.95)$ & 1 (reference) & 1 (reference) \\
\hline No & 1261 & 82.9 & 66 & 81.5 & $0.93(0.91-0.95)$ & $0.8(0.4-1.4)$ & $1.0(0.5-1.8)$ \\
\hline \multicolumn{8}{|l|}{ Hormone therapy } \\
\hline Yes & 738 & 48.5 & 16 & 19.8 & $0.94(0.90-0.97)$ & 1 (reference) & 1 (reference) \\
\hline No & 784 & 51.5 & 65 & 80.2 & $0.92(0.89-0.94)$ & $1.7(1.0-3.1)$ & $1.4(0.7-2.8)$ \\
\hline
\end{tabular}

${ }^{a}$ Hazard ratio adjusted for age (variable in continuous), period of diagnosis, method of discovery, grade, tumor size, surgery, radiotherapy, tumor location, histologic type, and hormone therapy; ${ }^{*} P<0.05,{ }^{*} P<0.01,{ }^{* * *} P<0.001 ;^{b}$ 9-year specific survival as information on estrogen receptors was available only from 1995.

TABLE 4. Distribution and effect of tumor location on breast cancer mortality among women operated for stage I breast cancer, Geneva Cancer Registry, 1984-2002

\begin{tabular}{lrrrrcrc}
\hline & \multicolumn{8}{c}{ Breast cancer mortality } \\
\cline { 2 - 8 } Patient distribution & $\mathrm{N}$ & $\%$ & $\mathrm{~N}$ of death & $\%$ & 10-year survival (95\%CI) & Age-adjusted HR (95\%CI) & Multiadjusted HR $^{a}(95 \% \mathrm{CI})$ \\
\hline Location & & & & & & & \\
Upper-outer quadrant & 588 & 38.6 & 25 & 30.9 & $0.94(0.91-0.96)$ & 1 (reference) & 1 (reference) \\
Upper-inner quadrant & 212 & 13.9 & 9 & 11.1 & $0.96(0.92-0.99)$ & $1.0(0.5-2.2)$ & $1.0(0.5-2.2)$ \\
Lower-outer quadrant & 118 & 7.8 & 7 & 8.6 & $0.91(0.85-0.98)$ & $1.3(0.6-3.0)$ & $1.3(0.6-3.1)$ \\
Lower-inner quadrant & 118 & 7.8 & 13 & 16.0 & $0.88(0.81-0.96)$ & $2.6^{* *}(1.3-5.0)$ & $0.3 *(1.1-4.5)$ \\
Centre, nipple & 54 & 3.5 & 2 & 2.5 & $0.94(0.87-1.00)$ & $0.8(0.2-3.6)$ & $0.6(0.1-2.8)$ \\
Overlapping quadrants & 413 & 27.1 & 19 & 23.5 & $0.93(0.89-0.96)$ & $1.1(0.6-2.1)$ & $1.1(0.6-2.0)$ \\
Unknown & 19 & 1.2 & 6 & 7.4 & $0.69(0.47-0.92)$ & - & - \\
\hline
\end{tabular}

${ }^{a}$ Hazard ratio adjusted for age (variable in continuous), period of diagnosis, method of discovery, grade, tumor size, surgery, radiotherapy, tumor location, histologic type, and hormone therapy; ${ }^{*} P<0.05,{ }^{* *} P<0.01$, ${ }^{* * *} P<0.001$.

Studies on the effect of tumor location on prognosis report inconsistent results. Some studies showed higher risks of death from breast cancer in patients with tumors of the inner quadrants, ${ }^{9,10,12}$ or inner quadrants plus central portion of the breast, ${ }^{11}$ or lower quadrants, ${ }^{24}$ or all quadrants except the upper- outer quadrant, ${ }^{25}$ whereas other studies showed no association whatsoever between tumor location and breast cancer prognosis. ${ }^{13-15,26}$

These inconsistencies could be explained by the fact that some studies that reported no association date from an era when women did not receive 
adjuvant chemotherapy in case of axillary or IMC lymph node metastases. ${ }^{4,14,15}$ It is impossible to evaluate an effect of undertreatment linked to understaging if node positive patients do not receive adjuvant treatment. One study did not observe increased breast cancer mortality but reported a significant increased risk of distant metastases in patients with inner quadrant tumors who did not receive chemotherapy. ${ }^{13}$ Longer follow-up will probably show an increased mortality risk among these patients.

Another explanation for the discrepancies between the studies is that most previous studies included patients with positive axillary lymph nodes. ${ }^{9-12,24,25,27,28}$ As these patients were already candidates for adjuvant therapy, the potential impact of undetected IMC metastases on survival was low. Among the few studies providing powerful subgroup analyses for lymph node negative patients, $, 12,25,29$ the majority reported a stronger effect of tumor location on breast cancer mortality among lymph node negative patients. ${ }^{9,25,29}$ Like our study, the recent study of Colleoni and colleagues reported that the effect of tumor location was greater for larger than for smaller tumours. ${ }^{9}$

As discussed above for the study of Veronesi and colleagues, the discrepancies between previous studies could also be explained by the dilution of the effect of the lower-inner quadrant, by regrouping this quadrant with other quadrants. ${ }^{4}$ Unfortunately, most studies reporting an association between breast location and survival did not distinguish between the two inner quadrants or the two lower quadrants, making it impossible to draw any conclusion for just the lower-inner quadrant. When we regrouped patients with tumors of the inner quadrants and compared them to patients with tumors of all other quadrants, as performed in previous studies, ${ }^{9,10,12}$ we found a similar mortality risk (HR: $1.3,95 \%$ CI: $0.8-2.2, P=0.241$ ). Also, when we regrouped patients with tumors of the lower quadrants and compared them to patients with tumors of all other quadrants, as performed in one other study, ${ }^{24}$ again we observed very comparable results (HR: 1.7, 95\%CI: $1.0-2.8, P=0.058$ ).

With the generalization of sentinel lymph node biopsy for early breast cancer, ${ }^{21,22,30}$ studies on the importance and consequences of IMC involvement among patients without axillary lymph node metastases become increasingly important. Systematic investigation of sentinel nodes of the IMC is feasible with an experienced team of professionals, even if difficulties exist in distinguishing between the radioactivity of the primary tumor located in the internal site of the breast and the radioactivity of the involved sentinel nodes in the IMC.

In conclusion, tumor location in the lower-inner quadrant is an independent and important prognostic factor for patients with node negative breast cancer. If further research demonstrates that this over-mortality is indeed due to a higher frequency of undetected IMC metastases, patients with stage I breast cancer of the lower-inner quadrant should become candidates for systematic evaluation of the IMC.

\section{ACKNOWLEDGMENTS}

Dr. H. M. Verkooijen was financially supported by PROSPER grant No. 3233-069350 from the Swiss National Science Foundation. We thank Ms S. Blagojevic for her editorial assistance.

\section{REFERENCES}

1. Sant M, Allemani C, Capocaccia R, et al. Stage at diagnosis is a key explanation of differences in breast cancer survival across Europe. Int J Cancer 2003; 106:416-22.

2. Cianfrocca M, Goldstein LJ. Prognostic and predictive factors in early-stage breast cancer. Oncologist 2004; 9:606-16.

3. Bouchardy C, Rapiti E, Fioretta G, et al. Undertreatment strongly decreases prognosis of breast cancer in elderly women. $J$ Clin Oncol 2003; 21:3580-7.

4. Veronesi U, Cascinelli N, Bufalino R, et al. Risk of internal mammary lymph node metastases and its relevance on prognosis of breast cancer patients. Ann Surg 1983; 198:681-4.

5. Veronesi U, Valagussa P. Inefficacy of internal mammary nodes dissection in breast cancer surgery. Cancer 1981; 47:170-5.

6. Cox EF, Buxton RW. Internal mammary lymph node biopsy as a guide to postmastectomy radiation therapy in breast carcinoma. Am Surg 1960; 26:335-40.

7. Dupont E, Cox CE, Nguyen K, et al. Utility of internal mammary lymph node removal when noted by intraoperative gamma probe detection. Ann Surg Oncol 2001; 8:833-6.

8. Shahar KH, Buchholz TA, Delpassand E, et al. Lower and central tumor location correlates with lymphoscintigraphy drainage to the internal mammary lymph nodes in breast carcinoma. Cancer 2005; 103:1323-9.

9. Colleoni M, Zahrieh D, Gelber RD, et al. Site of primary tumor has a prognostic role in operable breast cancer: the international breast cancer study group experience. $J$ Clin Oncol 2005; 23:1390-400.

10. Lohrisch C, Jackson J, Jones A, et al. Relationship between tumor location and relapse in 6,781 women with early invasive breast cancer. J Clin Oncol 2000; 18:2828-35.

11. Zucali R, Mariani L, Marubini E, et al. Early breast cancer: evaluation of the prognostic role of the site of the primary tumor. J Clin Oncol 1998; 16:1363-6.

12. Gaffney DK, Tsodikov A, Wiggins CL. Diminished survival in patients with inner versus outer quadrant breast cancers. J Clin Oncol 2003; 21:467-72.

13. Fowble B, Solin LJ, Schultz DJ, Weiss MC. Breast recurrence and survival related to primary tumor location in patients undergoing conservative surgery and radiation for early-stage breast cancer. Int J Radiat Oncol Biol Phys 1992; 23:933-9.

14. Fisher B, Wolmark N, Redmond C, et al. Findings from NSABP Protocol No. B-04: comparison of radical mastectomy with 
alternative treatments. II. The clinical and biologic significance of medial-central breast cancers. Cancer 1981; 48:1863-72.

15. Veronesi U, Cascinelli N, Greco M, et al. Prognosis of breast cancer patients after mastectomy and dissection of internal mammary nodes. Ann Surg 1985; 202:702-7.

16. Breslow NE, Day NE. Statistical methods in cancer research. Vol.II : the design and analysis of cohort studies. Lyon International Agency for Research on Cancer, IARC Scientific Publications no 82, 1987.

17. Lacour J, Bucalossi P, Cacers E, et al. Radical mastectomy versus radical mastectomy plus internal mammary dissection. Five-year results of an international cooperative study. Cancer 1976; 37:206-14.

18. Fisher B, Slack N, Katrych D, Wolmark N. Ten year follow-up results of patients with carcinoma of the breast in a co-operative clinical trial evaluating surgical adjuvant chemotherapy. Surg Gynecol Obstet 1975; 140:528-34.

19. Goldhirsch A, Wood WC, Gelber RD, et al. Meeting highlights: updated international expert consensus on the primary therapy of early breast cancer. J Clin Oncol 2003; 21:3357-65.

20. Recht A, Edge SB, Solin LJ, et al. Postmastectomy radiotherapy: clinical practice guidelines of the American Society of Clinical Oncology. J Clin Oncol 2001; 19:1539-69.

21. Freedman GM, Fowble BL, Nicolaou N, et al. Should internal mammary lymph nodes in breast cancer be a target for the radiation oncologist? Int J Radiat Oncol Biol Phys 2000; 46:805-14.
22. Estourgie SH, Tanis PJ, Nieweg OE, et al. Should the hunt for internal mammary chain sentinel nodes begin? An evaluation of 150 breast cancer patients. Ann Surg Oncol 2003; 10:935-41.

23. Estourgie SH, Nieweg OE, Olmos RA, et al. Lymphatic drainage patterns from the breast. Ann Surg 2004; 239:232-7.

24. Kamakura T, Akazawa K, Nomura Y, et al. Poor prognosis of lower quadrant breast carcinoma. Nishi Nippon Study Group on Adjuvant Chemo-endocrine Therapy for Breast Cancer. J Surg Oncol 1996; 61:295-9.

25. Kroman N, Wohlfahrt J, Mouridsen HT, Melbye M. Influence of tumor location on breast cancer prognosis. Int $J$ Cancer 2003; 105:542-5.

26. Boyages J, Recht A, Connolly JL, et al. Early breast cancer: predictors of breast recurrence for patients treated with conservative surgery and radiation therapy. Radiother Oncol 1990; 19:29-41.

27. Cody HS III, Urban JA. Internal mammary node status: a major prognosticator in axillary node-negative breast cancer. Ann Surg Oncol 1995; 2:32-7.

28. Nemoto T, Natarajan N, Bedwani R, et al. Breast cancer in the medial half Results of 1978 National Survey of the American College of Surgeons. Cancer 1983; 51:1333-8.

29. Nemoto T, Vana J, Bedwani RN, et al. Management and survival of female breast cancer: results of a national survey by the American College of Surgeons. Cancer 1980; 45:2917-24.

30. Noguchi M. Relevance and practicability of internal mammary sentinel node biopsy for breast cancer. Breast Cancer 2002; 9:329-36. 